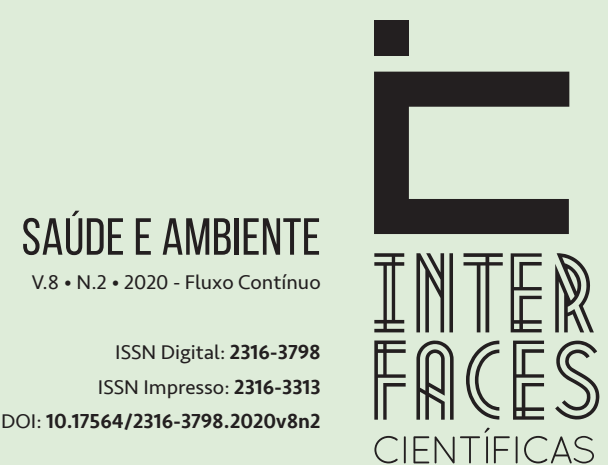

\section{CAPACIDADE FUNCIONAL E QUALIDADE DE VIDA DE IDOSOS PARTICIPANTES E NÃO PARTICIPANTES DE GRUPOS DE PROMOÇÃO DA SAÚDE}

\section{FUNCTIONAL CAPACITY AND QUALITY OF LIFE OF ELDERLY PARTICIPANTS AND NON-PARTICIPANTS OF HEALTH PROMOTION GROUPS}

\section{CAPACIDAD FUNCIONAL Y CALIDAD DE VIDA DE PARTICIPANTES MAYORES Y NO PARTICIPANTES DE GRUPOS DE PROMOCIÓN DE LA SALUD}

Regiane Vitorino Borges ${ }^{1}$ Tatiane Lima Teixeira da Silva ${ }^{2}$ Mateus Dias Antunes ${ }^{3}$ Sonia Maria Marques Gomes Bertolini ${ }^{4}$

Fernanda Shizue Nishida ${ }^{5}$ Aliny de Lima Santos ${ }^{6}$

\section{RESUMO}

Este estudo buscou analisar o impacto das atividades de promoção da saúde na capacidade funcional e qualidade de vida de idosos no âmbito da atenção primária. Trata-se de um estudo com abordagem quantitativa do tipo descritivo, desenvolvido com idosos cadastrados e acompanhados em uma Unidade Básica de Saúde do sul do país. As atividades de promoção da saúde eram desapega, bingo, pesqueiro e capoterapia. A coleta de dados se deu por meio de entrevista domiciliar semiestruturada com o uso dos seguintes questionários: escala de Katz e escala de Lawton Brody para a avaliação das atividades de vida diária, e WHOQOL-BREF para avaliação da qualidade de vida. Os dados foram registrados em instrumento impresso e analisados segundo estatística descritiva. Foram entrevistados 90 idosos, em dois grupos, sendo 45 idosos participantes de grupos de promoção da saúde (GP) e 45 idosos não participantes (GNP). Os idosos do GP se mostraram mais independentes nas atividades básicas $(p=0,018)$ e instrumentais $(p=0,015)$ na execução das atividades diárias e obtiveram diferença significativa $(p=0,002)$ da qualidade de vida quando comparados aos idosos do GNP. A partir dos dados achados foi possível concluir que as práticas de promoção da saúde estão relacionadas a uma melhor preservação da capacidade funcional e qualidade de vida.

\section{PALAVRAS-CHAVE}

Idosos, Qualidade de vida, Promoção da saúde, Atividades diárias, Atenção primária a saúde. 


\section{ABSTRACT}

This study aimed to analyze the impact of health promotion activities on the functional capacity and quality of life of the elderly in primary care. This is a study with quantitative descriptive approach, developed with elderly registered and followed in a Basic Health Unit of the south of the country. Health promotion activities were detachment, bingo, fishing and capotherapy. Data were collected through semi-structured home interviews using the following questionnaires: Katz scale and Lawton Brody scale to assess daily life activities, and WHOQOL-BREF to assess quality of life. Data were recorded in a printed instrument and analyzed according to descriptive statistics. Ninety elderly were interviewed in two groups, 45 elderly participants from health promotion groups (GP) and 45 elderly non-participants (GNP). The elderly in the GP were more independent in basic $(p=0.018)$ and instrumental $(p=0.015)$ activities in performing daily activities and had a significant difference $(p=0.002)$ in quality of life when compared to the elderly in GNP. From the data found, it was concluded that health promotion practices are related to better preservation of functional capacity and quality of life.

\section{KEYWORDS}

Elderly, Quality Of Life, Health Promotion, Daily Activities, Primary Health Care.

\section{RESUMEN}

Este estudio tuvo como objetivo analizar el impacto de las actividades de promoción de la salud en la capacidad funcional y la calidad de vida de los ancianos en atención primaria. Este es un estudio, con enfoque descriptivo cuantitativo, desarrollado con personas mayores registradas y seguidas en una Unidad Básica de Salud del sur del país, observando actividades de promoción de la salud. Los datos fueron recolectados a través de entrevistas domiciliarias semiestructuradas utilizando los siguientes cuestionarios: escala de Katz y escala de Lawton Brody para evaluar las actividades de la vida diaria, y WHOQOL-BREF para evaluar la calidad de vida. Los datos se registraron en un instrumento impreso y se analizaron de acuerdo con estadísticas descriptivas. Noventa ancianos fueron entrevistados en dos grupos, 45 participantes ancianos de grupos de promoción de la salud (GP) y 45 no participantes (GNP). Los ancianos en el GP fueron más independientes en actividades básicas $(p=0.018)$ e instrumentales $(p=0.015)$ en la realización de actividades diarias y tuvieron una diferencia significativa ( $p=0.002$ ) en la calidad de vida en comparación con los ancianos en el GNP. A partir de los datos encontrados, se concluyó que las prácticas de promoción de la salud están relacionadas con una mejor preservación de la capacidad funcional y la calidad de vida. 


\section{PALABRAS CLAVE}

Ancianos, Calidad de vida, Promoción de la salud, Actividades diarias, Atención primaria de salud.

\section{INTRODUCÇÃO}

O envelhecimento populacional vem se tornando cada vez mais significativo no mundo. No Brasil estima-se que a cada dez pessoas, uma tenha 60 anos ou mais, e em 2050 essa estimativa será de um a cada cinco brasileiros. Essa mudança demográfica vem levantando a questão sobre o cuidado a saúde da população idosa, seja no âmbito individual ou coletivo, a sociedade começa a se adaptar para garantir um envelhecimento saudável (FREIRE et al., 2015)

O envelhecer é um processo natural, pode não ser definido apenas pela perda da capacidade funcional e cognitiva, mas também por um conjunto de fatores individuais ao longo da vida da pessoa que influenciam na qualidade da sua velhice. Essa qualidade está relacionada a aspectos psicológicos, fisiológicos, sociais, culturais e econômicos que contribuem de forma estrutural para a autonomia funcional e interação social do idoso (BRAGA et al., 2015).

Nos idosos a capacidade funcional pode ser entendida como o potencial que estes apresentam para atuarem com autonomia e independência em suas atividades diárias (TELES et al., 2017). A incapacidade em realizar as Atividades Básicas de Vida Diária (ABVD) e as Atividades Instrumentais de Vida Diária (AIVD), gera nele um maior risco de mortalidade, hospitalização, dependência e alto gasto com os serviços de saúde (CORDEIRO et al., 2014).

Como estratégia de cuidado para os idosos, a Atenção Primária a Saúde (APS) instrumentaliza ações de acordo com a Política Nacional de Saúde da Pessoa Idosa (PNSPI), que em seus princípios busca manter a autonomia e independência deles em seu contexto social e familiar por meio da manutenção da funcionalidade, promoção do envelhecimento ativo e saudável, prevenção e recuperação da saúde (MARANDI et al., 2017).

Diversas ações que visam a promoção da saúde (PS) têm como objetivos propiciar autonomia aos indivíduos para exercer o controle de sua saúde e vida por meio da participação em atividades coletivas e grupais (OLIVEIRA et al., 2017). Sendo assim, as atividades em grupo se configuram como ferramentas utilizadas para recuperação e manutenção de incapacidades físicas e cognitivas dos indivíduos participantes, além de oferecer bem-estar, autonomia e qualidade de vida, contribuindo também para a inclusão e interação social, lazer, e adoção de um estilo de vida ativo e saudável (MACHADO et al., 2017).

Estudo realizado com idosos participantes de um grupo de convivência, mostrou índice elevado de qualidade de vida, evidenciando que apesar dos declínios funcionais inerentes ao envelhecimento, os que se mantêm ativos e participativos no convívio social podem envelhecer de forma mais saudável, preservando sua funcionalidade (SANTOS et al., 2015). 
Desse modo, acredita-se que a oferta de atividades de promoção à saúde no âmbito da Atenção Primária possa favorecer a funcionalidade, mobilidade e qualidade de vida para os idosos. Diante disso, o objetivo do estudo foi analisar a capacidade funcional na execução das ABVD e AIVD e a qualidade de vida de idosos participantes e não participantes dos grupos de PS.

\section{MÉTODO}

Trata-se de um estudo quantitativo do tipo descritivo que foi realizado com idosos cadastrados na Equipe Saúde da Família (ESF) de uma Unidade Básica de Saúde (UBS) de Maringá, localizada na região norte do Paraná. Esta UBS conta com duas ESF que atendem um total de 1.839 participante idosos. A escolha da unidade e dos participantes do estudo foi por conveniência.

Foram incluídos no estudo indivíduos com a faixa etária de 60 anos ou mais, cadastrados e assistidos na UBS elencada, que receberam pontuação adequada no Mini-exame do Estado Mental e classificados pela unidade como robustos, segundo estratificação de idosos para a Rede de Atenção à Saúde do Idoso.

Os idosos foram divididos em dois grupos, aqueles que participam (GP) e aqueles que não participam (GNP) de atividades de promoção da saúde realizadas na UBS. Inicialmente, por meio de uma lista disponibilizada pelas ESF da unidade, foram identificados 1.030 idosos robustos dos quais 55 participavam dos grupos de promoção da saúde. Para o GP foram convidados todos os da terceira idade que participam das atividades de PS realizadas na UBS, sendo que 45 aceitaram participar do estudo. 0 convite e a explicação de como se daria a participação aconteceu na Atenção Primária.

As atividades de Promoção da Saúde oferecidas pela UBS são realizadas em quatro modalidades, sendo que três são mensais (desapega, bingo e pesqueiro) e uma semanal (capoterapia). A capoterapia utiliza movimentos básicos da capoeira adaptados para a população idosa e os demais grupos propiciam a socialização por meio de atividades de entretenimento.

Com intuito de equiparar os grupos, optou-se por inserir também 45 idosos GNP, sendo composto por não participantes das atividades. A escolha ocorreu, por meio de um sorteio aleatório, utilizando a lista de idosos robustos não participantes, fornecida pela UBS (FIGURA 1). Para o segundo grupo, foram realizadas visitas domiciliares para convite quanto à participação da pesquisa, sendo substituídos pelo próximo sorteado da lista em caso de recusa ou ainda após três tentativas de contato sem sucesso, até obter o número de participantes igual ao outro grupo. 
Figura 1 - Fluxograma da seleção dos idosos

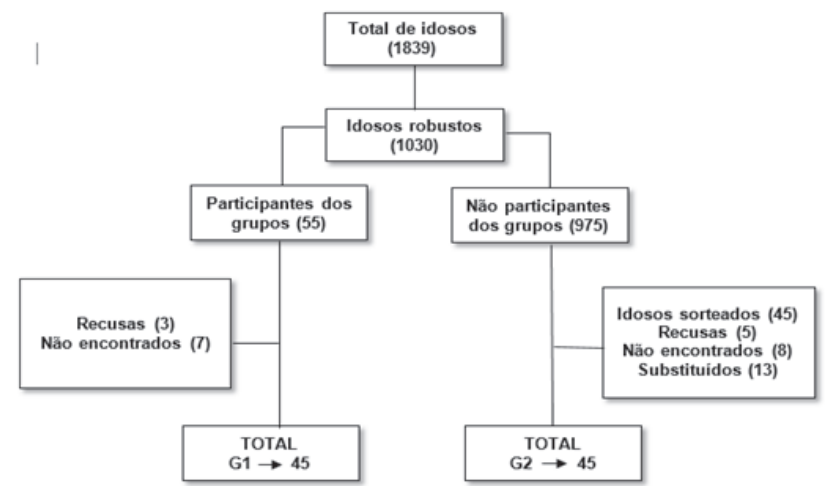

Fonte: Os autores.

O estudo foi realizado no período de julho a agosto de 2018, por meio de visitas domiciliares previamente agendadas com o auxílio de uma Agente Comunitária de Saúde (ACS), de modo a não comprometer as entrevistas ou direcionar as respostas.

Os dados foram obtidos por meio de entrevista semiestruturada e foram utilizados três instrumentos de avaliação (Escala de Katz, Escala de Lawton e Brody e WHOQOL- BREF).

Para avaliação da funcionalidade foram utilizadas duas escalas, a escala de Katz que avalia as ABVDs como tomar banho, se vestir, ir ao banheiro, se locomover, controle de eliminações e alimentação. E a Lawton e Brody para AIVD que envolve conseguir cuidar da casa, lavar roupa, cozinhar, fazer compras, utilizar telefone, usar meios de transporte, uso do dinheiro e responsabilidade sobre medicações (APÓSTOLO, 2012).

Já para avaliação da qualidade de vida, foi utilizado o WHOQOL- BREF, instrumento da Organização Mundial de Saúde (OMS), validado e traduzido para o português com a finalidade de avaliar a qualidade de vida por meio de 26 questões (FLECK et al., 2000). As duas primeiras questões têm caráter geral e as outras 26 representam as facetas do instrumento original que avaliam quatro domínios:

Físico (dor e desconforto, energia e fadiga, sono e repouso, mobilidade, atividades da vida cotidiana, dependência de medicação ou de tratamentos e capacidade de trabalho);

Psicológico (sentimentos positivos, pensar, aprender, memória e concentração, autoestima, imagem corporal e aparência, sentimentos negativos, espiritualidade, religião e crenças pessoais);

Relações Sociais (relações pessoais, suporte social e atividade sexual);

Meio Ambiente (segurança física e proteção, ambiente no lar, recursos financeiros, cuidados de saúde e sociais: disponibilidade e qualidade, oportunidade de adquirir novas informações e habilidades, participação/oportunidades de recreação/lazer, ambiente físico: poluição, ruído, trânsito, clima e transporte) (FLECK et al., 2000).

O WHOQOL-bref é pontuado no formato Likert, que mede frequência, intensidade, capacidade e avaliação, sendo que as respostas de cada questão variam de um a cinco. 0 escore médio dos do- 
mínios indica a percepção dos idosos sobre a qualidade de vida por meio da avaliação de diferentes aspectos. Quanto maior a pontuação, melhor essa percepção (FLECK et al., 2000).

Os dados foram apresentados por meio da estatística descritiva (média, desvio padrão, frequências absoluta e relativa, gráficos e tabelas). Para análise dos resultados foi aplicado o teste t Student com nível de significância de $5 \%(p<0,05)$.

A pesquisa seguiu todos os preceitos da Resolução que viabiliza pesquisas com seres humanos, de $n^{\circ}$ 466, de 12 de dezembro de 2012, do Conselho Nacional de Saúde, bem como apreciação e aprovação do Comitê de Ética em Pesquisa do Centro Universitário de Maringá - UNICESUMAR (CAAE: 90795418.4.0000.5539). Foi utilizado o Termo de Consentimento Livre e Esclarecido em duas vias, ficando uma com os pesquisadores e uma com os idosos participantes.

\section{RESULTADOS}

Em ambos os grupos houve predominância do sexo feminino, tinham idade entre 60 a 69 anos, apresentavam até 4 anos de estudo e apresentaram doenças crônicas (TABELA 1).

Tabela 1 - Perfil socioeconômico e de saúde de idosos participantes e não participantes de grupos de promoção a saúde oferecidos na UBS Parigot de Souza de Maringá-PR, 2018

\begin{tabular}{|c|c|c|c|c|}
\hline \multirow{2}{*}{ Variáveis } & \multicolumn{2}{|c|}{ GP } & \multicolumn{2}{c|}{ GNP } \\
\cline { 2 - 5 } & $\mathbf{N}$ & $\mathbf{N}$ & $\mathbf{N}$ & \\
\hline Gênero & & & 33 & 73,3 \\
\hline Feminino & 37 & 82,2 & 12 & 26,7 \\
\hline Masculino & 8 & 17,8 & & \\
\hline Idade & & & 21 & 46,7 \\
\hline $60-69$ & 21 & 46,7 & 18 & 40,0 \\
\hline $70-79$ & 20 & 44,4 & 6 & 13,3 \\
\hline $80-89$ & 4 & 8,9 & & \\
\hline Escolaridade (anos de estudo completos) & 6 & 13,3 & 17 & 37,8 \\
\hline Não estudou & 32 & 71,1 & 24 & 53,3 \\
\hline Até 4 anos de estudo & 7 & 15,5 & 4 & 8,9 \\
\hline 4 anos ou mais & \multicolumn{2}{|c}{} & & \\
\hline Estado civil & 5 & 11,1 & 2 & 4,4 \\
\hline Solteiro & & & & \\
\hline
\end{tabular}




\begin{tabular}{|c|c|c|c|c|}
\hline \multirow{2}{*}{ Variáveis } & \multicolumn{2}{|c|}{ GP } & \multicolumn{2}{|c|}{ GNP } \\
\hline & $\mathbf{N}$ & $\%$ & $\mathbf{N}$ & $\%$ \\
\hline Casado & 22 & 48,9 & 24 & 53,3 \\
\hline Outros & 18 & 40 & 19 & 42,2 \\
\hline \multicolumn{5}{|l|}{ Aposentado ou pensionista } \\
\hline Sim & 35 & 77,8 & 35 & 77,8 \\
\hline Não & 10 & 22,2 & 10 & 22,2 \\
\hline \multicolumn{5}{|l|}{ Renda familiar } \\
\hline 1 salário mínimo & 20 & 44,4 & 17 & 37,8 \\
\hline 2 salários mínimos & 20 & 44,4 & 20 & 44,4 \\
\hline 3 ou mais salários mínimos & 5 & 11,1 & 8 & 17,8 \\
\hline \multicolumn{5}{|l|}{ Convive com familiares } \\
\hline Sim & 39 & 86,7 & 32 & 71,1 \\
\hline Não & 6 & 13,3 & 13 & 28,9 \\
\hline \multicolumn{5}{|c|}{ Presença de doença Crônica } \\
\hline Sim & 38 & 84,4 & 42 & 93,3 \\
\hline Não & 7 & 15,5 & 3 & 6.7 \\
\hline \multicolumn{5}{|l|}{ Medicamentos em uso } \\
\hline Não usa & 6 & 13,3 & 4 & 8,9 \\
\hline $1-2$ & 25 & 55,5 & 20 & 44,4 \\
\hline 3 ou mais & 14 & 31,1 & 21 & 46,7 \\
\hline \multicolumn{5}{|l|}{ Prática de atividade física } \\
\hline Sim & 35 & 77,7 & 14 & 31,1 \\
\hline Não & 10 & 22,2 & 31 & 68,9 \\
\hline \multicolumn{5}{|l|}{ Auto percepção de saúde } \\
\hline Excelente - ótima & 9 & 20,0 & 10 & 22,2 \\
\hline Boa & 22 & 48,9 & 15 & 33,3 \\
\hline Razoável - péssima & 14 & 31,1 & 20 & 44,4 \\
\hline
\end{tabular}

Legenda: GP: Grupo de participantes; GNP: Grupo de não participantes.

Fonte: Os autores. 
Em relação à funcionalidade, verificou-se que os idodos de ambos os grupos se encontravam em situação de independência para realizar ABVD sem a necessidade de supervisão ou auxílio (TABELA 2).

Tabela 2 - Classificação da funcionalidade de idosos participantes e não participantes de grupos de promoção a saúde em relação as Atividades Básicas e Instrumentais de Vida Diária

\begin{tabular}{|c|c|c|c|c|c|c|}
\hline \multirow{2}{*}{ Variáveis } & \multirow{2}{*}{ Categorias } & \multicolumn{2}{|c|}{ GP } & \multicolumn{2}{|c|}{ GNP } & \multirow{2}{*}{ Valor de $p$} \\
\hline & & $\mathbf{N}$ & $\%$ & $\mathbf{N}$ & $\%$ & \\
\hline \multirow{2}{*}{ ABVD } & Independente & 45 & 100 & 42 & 93,3 & \\
\hline & Algum tipo de dependência & - & - & 3 & 6,7 & $0,018^{*}$ \\
\hline \multirow{2}{*}{ AIVD } & Independente & 33 & 73,3 & 22 & 48,9 & \\
\hline & Algum tipo de dependência & 12 & 26,7 & 23 & 51,1 & $0,015^{*}$ \\
\hline
\end{tabular}

*valor de significância p<0,05. Legenda: GP: Grupo de participantes; GNP: Grupo de não participantes. Legenda: ABVDs: Atividades Básicas de Vida Diária; AIVDs: Atividades Instrumentais de Vida Diária. Fonte: Os autores.

No aspecto relativo à avaliação da qualidade de vida, o domínio mais prevalente foi relações sociais, seguido pelo domínio qualidade de vida global e meio ambiente (TABELA 3).

Tabela 3 - Percepção da qualidade de vida dos idosos participantes e não participantes dos grupos de promoção a saúde conforme pontuação dos domínios referentes ao instrumento WHOQOL-BREF

\begin{tabular}{|c|c|c|c|}
\hline \multirow{2}{*}{ Domínios } & GP & GNP & \multirow{2}{*}{ Valor de $p$} \\
\cline { 2 - 3 } & $\begin{array}{c}\text { Média } \pm \\
\text { Desvio Padrão }\end{array}$ & $\begin{array}{c}\text { Média } \pm \\
\text { Desvio Padrão }\end{array}$ & \\
\hline Físico & $15,61 \pm 1,65$ & $14,22 \pm 2,17$ & $0,005^{*}$ \\
\hline Psicológico & $15,42 \pm 1,56$ & $14,39 \pm 2,03$ & $0,024^{*}$ \\
\hline Relações Sociais & $16,42 \pm 1,51$ & $14,87 \pm 2,01$ & $0,001^{*}$ \\
\hline Meio Ambiente & $14,73 \pm 1,90$ & $13,49 \pm 2,00$ & $0,017^{*}$ \\
\hline Qualidade de Vida Global & $15,55 \pm 2,39$ & $14,62 \pm 2,44$ & 0,147 \\
\hline TOTAL & $15,38 \pm 1,47$ & $14,14 \pm 1,62$ & $0,002^{*}$ \\
\hline
\end{tabular}

* valor de significância p<0,05. Legenda: GP: Grupo de participantes; GNP: Grupo de não participantes. Fonte: Os autores.

Em relação às questões do WHOQOL-BREF dos GP, as opções relacionas a dor e desconforto $(23,8 \%)$, dependência de medicação ou e tratamentos $(27,2 \%)$ e sentimentos negativos estão mais comprometidos (FIGURA 2). 
Figura 2 - Médias dos escores das questões (facetas) do WHOQOL-BREF dos idosos participantes dos grupos de promoção a saúde

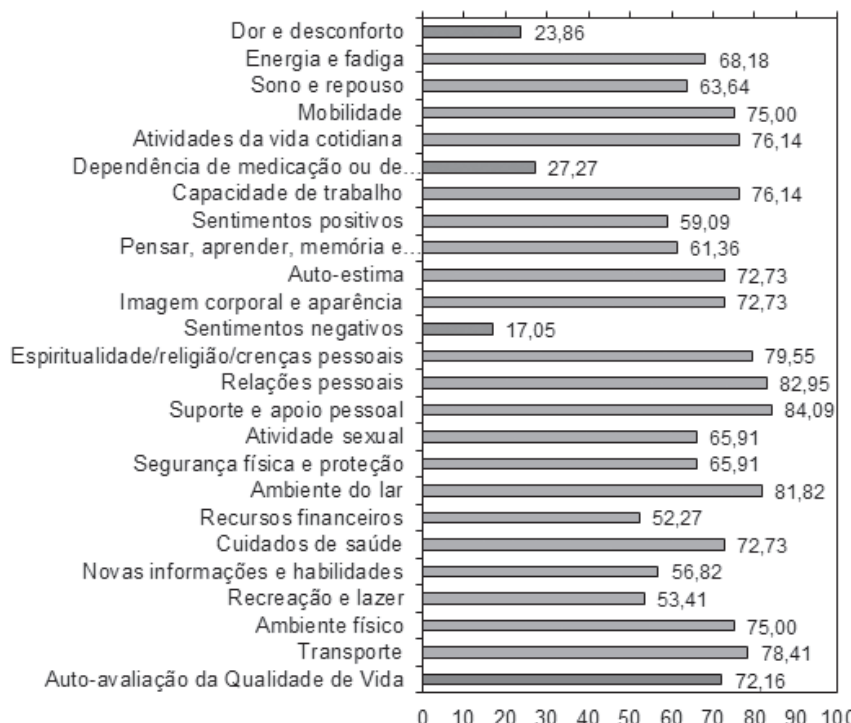

Fonte: Os autores.

Já o GNP, no WHOQOL-BREF as mesmas opções foram as mais comprometidas (FIGURA 3).

Figura 3 - Médias dos escores das questões (facetas) do WHOQOL-BREF dos idosos não participantes dos grupos de promoção a saúde

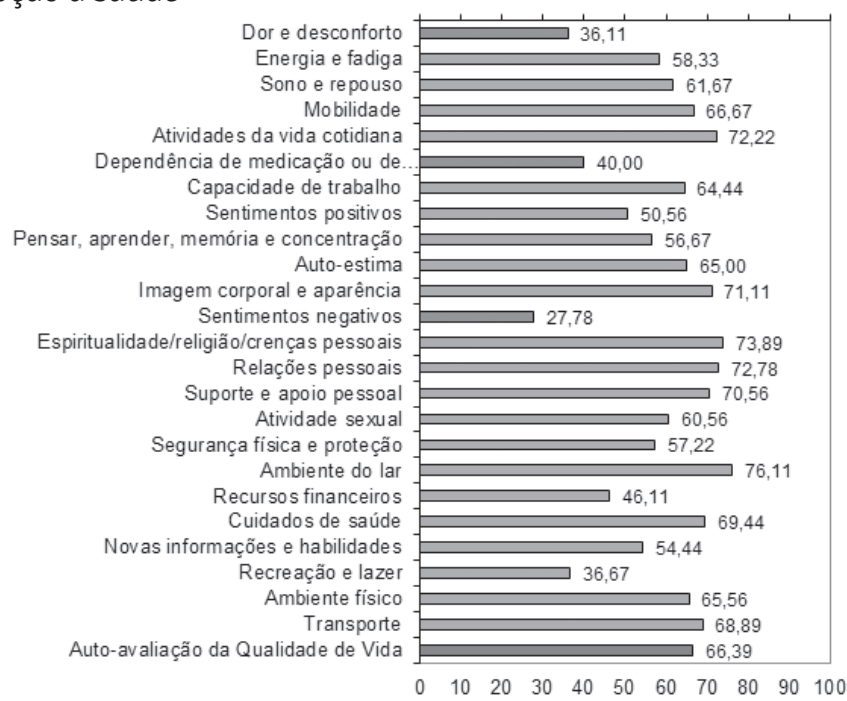

Fonte: Os autores. 


\section{DISCUSSÃO}

Foi constatada a predominância do sexo feminino em ambos os grupos, sendo ainda maior no primeiro grupo. Observa-se que as mulheres têm maior interesse na prática de atividades relacionadas à promoção a saúde, talvez por preocuparem-se mais com a saúde e com a adoção do estilo de vida saudável, se comparadas aos homens (SANTOS et al., 2015). Essa tendência de buscar uma melhor qualidade de vida aumenta a expectativa de vida feminina, o que torna este gênero predominante em estudos do tipo (SANTOS et al., 2015; FREIRE et al., 2015).

De modo geral, os indivíduos entrevistados são na maioria idosos jovens (60 a 79 anos) e com escolaridade baixa. Sabe-se que o nível de instrução é relevante para o desenvolvimento das habilidades sociais e a sua falta pode prejudicar até a saúde da população, pois interfere na compreensão e na percepção de saúde, tornando a baixa escolaridade um fator preocupante para o processo saúde-doença(MACHADO et al., 2017).

Em relação ao estado civil, houve um maior número de idosos casados, resultado este que não corresponde com o perfil exposto em estudo de Machado e colaboradores (2017) realizado com idosos de um grupo de convivência vinculado a um Centro de Saúde da Família (CSF) de um município do Nordeste brasileiro que também apresentou predominância do sexo feminino, mas evidenciou maior percentual de viúvas, justificando-se talvez pela maior expectativa de vida das mulheres.

A renda predominante foi a aposentadoria, com porcentagem maior de até dois salários mínimos mensais nos dois grupos. A aposentadoria traz algumas mudanças para a rotina do idoso, que deixa a vida ativa do trabalho e busca o descanso e lazer, no entanto, pode trazer sentimento de tristeza e incapacidade (FREIRE et al., 2015).

O convívio familiar foi uma característica presente nos idosos entrevistados, visto que, a maioria reside com algum familiar, o que nem sempre está atrelado a uma interação social satisfatória dentro de seu círculo familiar. Acredita-se que tal aspecto pode estimular o interesse dos idosos em participar das atividades de PS, por acreditarem que os mesmos podem proporcionar uma maior interação com o meio social (ARAÚJO et al., 2017).

No que se refere a aspectos da saúde, grande porcentagem dos idosos demonstraram prevalência de doenças crônicas, sendo que, no GNP apenas três idosos não apresentaram tal condição. Diante disso, a existência de doenças crônicas reflete um estilo de vida não saudável que compromete a vida e o bem-estar do idoso. Esses dados confirmam a necessidade de ações voltadas à manutenção da saúde dessa população, pontuando o compromisso de promover a melhora da qualidade de vida dos mesmos (MACHADO et al., 2017).

Com isso, o uso de medicamentos é uma característica muito presente. No GNP, a maioria faz uso de 3 ou mais medicamentos. Estudo aponta que de todas as medicações prescritas para a população brasileira, 50\% são para os idosos, fato relacionado ao maior índice de prevalência de doenças crônicas que atingem essa classe, fazendo com que eles sejam usuários constantes de medicamentos e dos serviços de saúde no Brasil (MACHADO et al., 2017).

Em relação à prática de atividade física, os idosos do GP se mostraram mais ativos, sendo a caminhada uma das atividades mais citadas por eles. 0 exercício físico é de suma importância para a 
qualidade de vida, pois auxiliam na prevenção e no tratamento de doenças crônicas, e ainda, atuam minimizando o declínio da capacidade funcional e mental. Ademais, pode ser vista como ferramenta no processo saúde-doença, contribuindo para a redução dos gastos públicos na medicina curativa (FREIRE et al., 2015).

Quanto à percepção de saúde, quando questionados, a maior parte dos idosos do GP a avaliaram como “boa”, e do GNP como "razoável ou péssima”. Estes resultados podem ser relacionados ao grau de dependência na execução de atividades, tendo em vista que em ambos os grupos, mesmo que maior no segundo houve prevalência de doenças crônicas. Valer e colaboradores (2015) observaram que os idosos relacionam a saúde à independência, sendo a capacidade funcional um importante indicador de saúde pela própria concepção dos idosos, que mesmo com a presença de doenças influenciam sua percepção de saúde a sua funcionalidade.

O resultado encontrado sobre a percepção de saúde remete a importância de atividades que representem um envelhecimento ativo e saudável aos idosos. A participação em grupos de PS surge como opção favorável para essas condições, pois, facilita, sobretudo, a manutenção da capacidade funcional, proporcionando a autonomia e independência na execução de suas atividades, o que representa uma das categorias do significado de "saúde" para esta população (VALER et al., 2015).

$\mathrm{Na}$ avaliação das ABVD, foi observado que os idosos GP mostraram-se independentes em sua totalidade. Perfil semelhante foi encontrado em um estudo com idosos assistidos pela ESF que frequentavam grupos com o objetivo principal de manter a população ativa (MARANDI et al., 2017). Com isso, é notório que os grupos os beneficiam na manutenção da mobilidade e funcionalidade e, além disso, favorecem adoção de um estilo de vida saudável, potencializam a socialização e a mobilidade física, despertando a satisfação consigo mesmo, minimizando tristezas e isolamento (MACHADO et al., 2017).

Já em relação aos participantes do GNP, foram encontrados indivíduos com algum nível de dependência na execução das mesmas atividades. A ausência de manutenção da saúde propicia o declínio funcional inerente ao envelhecimento, fato observado nesse achado. Sabidamente, a prática de exercícios físicos ou atividade em grupo se estabelece como uma medida capaz de postergar a alteração dos declínios funcionais e minimiza ainda o surgimento de enfermidades crônicas em idosos saudáveis (CORDEIRO et al., 2014).

No que diz respeito às AIVD, os idosos do GP também se mostraram mais independentes. A autonomia e a independência para a execução de tais atividades dependem do bom funcionamento de fatores como a mobilidade, cognição, comunicação e humor. Consequentemente, ao participarem dos grupos de PS eles promovem a manutenção desses fatores, sendo beneficiados com um maior índice de independência na execução dessas atividades (TELES et al., 2017).

As AIVD exigem maior capacidade cognitiva para execução, sendo que os idosos perdem primeiro a capacidade de realizar essas atividades, antes de surgir a dependência das atividades básicas. Contudo, os idosos do GNP demonstraram dependência consideravelmente maior na execução das AIVD, além de ser o grupo que apresentou dependência nas ABVD.

Esse achado pode estar relacionado à falta de manutenção da funcionalidade, que propicia o de- 
clínio contínuo das funções afetadas pelo envelhecimento. Além disso, a incapacidade de executar essas atividades causa inúmeros transtornos, prejudicando o convívio social e até a família, pois necessitam de auxílio e demanda maior mobilização para a execução (TELES et al., 2017).

Na avaliação da qualidade de vida, os escores de cada domínio do WHOQOL-BREF foram satisfatórios. O Domínio Físico obteve diferença significativa entre os grupos. Nota-se que os grupos de PS têm contribuição positiva sobre aspectos físicos dos idosos mesmo com as mudanças advindas do envelhecimento, tendo em vista que, durante as entrevistas foi observado pelos pesquisadores que alguns indivíduos do GNP mantinham respostas positivas em relação a este domínio mesmo sendo visível a dificuldade de locomoção, dor e o maior nível de dependência apresentado por eles na avaliação das atividades de vida diária. Tal comportamento pode estar relacionado à falta de capacitação e educação em saúde, o que prejudica o indivíduo a ter uma visão ampla no reconhecimento dos aspectos que afetam a independência e a saúde na velhice (SANTOS et al., 2015).

Em relação ao domínio psicológico, o GP obteve a maior média e também apresentando diferença significativa e de acordo com Souza e colaboradores (2018), que em seu estudo, identificaram maior chance de qualidade de vida ruim nesse aspecto em idosos com dependências funcionais, visto que, a incapacidade funcional afeta a autonomia e o sentimento de utilidade na vida.

No domínio Relações Sociais, o GP apresentou diferença estatística em relação ao GNP. Tal achado pode evidenciar que a participação em atividades de PS proporciona o desenvolvimento das relações sociais, fortalecendo o convívio social e novos relacionamentos interpessoais (COSTA et al., 2018). Nota-se aqui a importância de medidas que integrem o idoso ao meio social, mantendo-os engajados socialmente, fomentando seu sentimento de utilidade e assim, colaborando para a melhora da qualidade de vida (PEREIRA et al., 2018).

O domínio meio ambiente do WHOQOL-BREF diz respeito à segurança do indivíduo, questões financeiras e acesso a recursos de saúde, transporte e lazer. Neste domínio, o GP apresentou diferença digniticativa quando comparado ao GNP, sendo assim, podemos destar que as questões como a maior independência física encontrada nesses idosos influenciam o bom desempenho nas facetas desse domínio, pois possuem maior facilidade de deslocamento para garantir acesso aos recursos de lazer e saúde e ainda autonomia para atuarem como cidadãos ativos (SOUZA et al., 2018).

Contudo, foi evidenciado significativamente uma melhor qualidade de vida no escre total do instrumento utilizado. É evidente que a preservação da saúde física, o sentimento de segurança, reconhecimento e a oportunidade de participação e convívio no meio social contribuem para o envelhecimento com saúde e melhora a percepção das condições de vida. Pois, estes aspectos, possibilitam ao idoso ter contato com o meio externo a sua família e a atuar sobre sua saúde com autonomia (PEREIRA et al., 2018).

A qualidade de vida dos idosos do GP significativamente melhor. De modo geral, observamos que os idosos que se mantêm ativos fisicamente e no convívio social, participando dos grupos de PS se mostraram mais independentes em relação aos que não participam, evidenciando assim que tais atividades trazem benefícios a esta população que cresce progressivamente e necessita de ações de saúde que minimizem as incapacidades associadas àpartici velhice. 


\section{CONCLUSÃO}

O estudo permitiu conhecer a influência das atividades de PS na percepção dos idosos e comparar os dados encontrados entre os que participam regularmente de tais atividades e os que não participam. Verificou-se nesse contexto que os indivíduos que participam dos grupos e estão inseridos no meio social, engajados na manutenção da saúde física, mental e cognitiva são mais independentes e se percebem com melhor qualidade de vida diante do envelhecimento.

Já os resultados em relação aos idosos que não participam demonstraram maiores declínios funcionais e maior chance de qualidade de vida ruim, conforme a própria percepção dos indivíduos, evidenciando que a falta de práticas de PS se torna prejudicial ao envelhecimento saudável. Destarte, ressalta-se a importância dos grupos de promoção da saúde que vêm estimulando a população idosa a um estilo de vida saudável e ativo, promovendo a autonomia e independência, facilitando a socialização e proporcionado o bem-estar físico e mental.

A temática desta pesquisa é relevante diante do aumento da expectativa de vida que demanda maior necessidade de promover a qualidade de vida dos idosos. E ainda, os dados mais recentes contribuem para informações mais próximas da realidade que podem direcionar as ações dos profissionais envolvidos com esse público.

\section{REFERÊNCIA}

APÓSTOLO, J. Instrumentos para avaliação em geriatria. Documento de Apoio, v. 1, p. 1-13, 2012.

ARAÚJO, L. S. A. et al. Idosos e grupos de convivência: motivos para a não adesão. SANARE: Rev. Pol. Públ., v. 16, n. 1, p. 58-67, 2017.

BRAGA, I. B. et al. A percepção do Idoso sobre a Saúde e Qualidade de Vida na Terceira Idade. Rev. Multidiscipl. Psicol., v. 9, n. 26, p. 211-222, 2015.

CORDEIRO, J. et al. Efeitos da atividade física na memória declarativa, capacidade funcional e qualidade de vida em idosos. Rev. Bras. Geriatr. Gerontol., v. 17, n. 3, p. 541-552, 2014.

COSTA, I. P.et al. Qualidade de vida de idosos e sua relação com o trabalho. Rev. Gaúcha Enferm., v. 39, n. 1, p. 1-9, 2018.

FLECK, M. et al. Aplicação da versão em português do instrumento abreviado de avaliação da qualidade de vida” WHOQOL-bref”. Rev. Saúde Públ., v. 34, p. 178-183, 2000. 
FREIRE, G. V. et al. Perfil de idosos que frequentam um centro de convivência da terceira idade. Rev. Interdiscipl., v. 8, n. 2, p. 11-19, 2015.

MACHADO, W. D. et al. Idosos com doenças crônicas não transmissíveis: um estudo em grupos de convivência. Rev. Ciên. Saberes - Facema, v. 3, n. 2, p. 445-451, 2017.

MARANDI, B. A. N. et al. Avaliação da capacidade funcional de idosos: atividades das equipes da estratégia de saúde da família. Rev. Pesq: Cuidado é Fundamental, v. 9, n. 4, p. 1087-1093, 2017.

OLIVEIRA, F. A. et al. Estratégias educativas para promoção da saúde de idosos de um centro de convivência. Rev. Conexão UEPG, v. 13, n. 3, p. 500-511, 2017.

PEREIRA, F. N. et al. Impactos de intervenções com atividades físicas e psicossociais na qualidade de vida de pessoas idosas. Rev. Pesq. Práticas Psicossoc., v. 13, n. 1, p. 1-12, 2018.

SANTOS, L. F. et al. Qualidade de vida de idosos que participam de grupo de promoção da saúde. Enfermería Global, v. 14, n. 4, p. 1-32, 2015.

SOUZA, A. A. D. et al. Qualidade de vida e incapacidade funcional entre idosos cadastrados na estratégia de saúde da família. ABCS Health Sci., v. 43, n. 1, p. 24-24, 2018.

TELES, M. A. B. et al. Avaliação da capacidade funcional de idosos cadastrados em uma estratégia saúde da família. Rev. Enferm. UFPE, v. 11, n. 6, p. 2620-2627, 2017.

VALER, D. B. et al. O significado de envelhecimento saudável para pessoas idosas vinculadas a grupos educativos. Rev. Bras. Geriatr. Gerontol., v. 18, n. 4, p. 809-819, 2015. 
1 Enfermeira, Centro Universitário de Maringá, PR.

E-mail: regiane@hotmail.com

2 Enfermeira, Centro Universitário de Maringá, PR.

E-mail: tatilimadts@gmail.com

3 Mestre em Promoção da Saúde; Doutorando em Ciências da Reabilitação, Universidade de São Paulo, SP; Fisioterapeuta. E-mail: mateusantunes@usp.br

4 Doutora e Mestre em Ciências Morfofuncionais; Fisioterapeuta; Coordenadora do Programa de Pós-Graduação em Promoção da Saúde, Centro Universitário de Maringá, PR; Pesquisadora do Instituto Cesumar de Ciência, Tecnologia e Inovação - ICETI.

E-mail: sonia.bertolini@unicesumar.edu.br

5 Doutora em Enfermagem; Enfermeira; Professora do Programa de Pós-Graduação em Promoção da Saúde e do curso de Medicina do Centro Universitário de Maringá, PR; Pesquisadora do Instituto Cesumar de Ciência, Tecnologia e Inovação - ICETI.

Email: fernanda.nishida@unicesumar.edu.br

6 Doutora e Mestre em Enfermagem; Enfermeira; Professora do curso de Graduação em Medicina e Graduação em Enfermagem, Centro Universitário de Maringá, PR.

E-mail: aliny.lima.santos@gmail.com

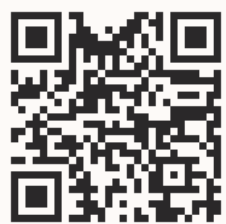

A autenticidade desse artigo pode ser conferida no site https://periodicos. set.edu.br

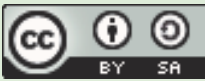

Este artigo é licenciado na modalidade acesso abertosob a Atribuição-Compartilhalgual CC BY-SA

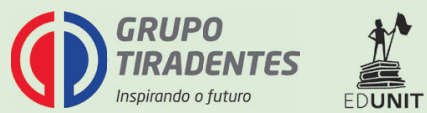


